DEMONSTRATIO MATHEMATICA

Vol. XXIX No $2 \quad 1996$

Nguyen Van Mau, Nguyen Minh Tuan

\title{
CHARACTERIZATIONS OF POLYNOMIALS IN RIGHT INVERTIBLE OPERATORS
}

In this paper we introduce the so-called $r$-Volterra operators and generalize the known-result of Przeworska-Rolewicz and von Trotha on Volterra characterizations of polynomials with scalar coefficients to the case of polynomials in algebraic elements in terms of $r$-Volterra characterizations.

Let $X$ be a linear space over the field of complex numbers. The set of all linear operators acting in $X$ is denoted by $L(X)$. Write: $L_{0}(X)=$ $\{A \in L(X): \operatorname{dom} A=X\}$. An operator $D \in L(X)$ is said to be right invertible if there exists an $R \in L_{0}(X)$ such that $R X \subset \operatorname{dom} D$ and $D R=I$. Denote by $R(X)$ the set of all right invertible operators and by $R_{D}$ the set of all right inverses of a $D \in R(X)$ (cf. [1]).

Recall the following results of Przeworska-Rolewicz and von Trotha [1]-[2]:

Theorem 1 (Przeworska-Rolewicz). Write

$$
\bar{Q}(t, s)=\sum_{k=0}^{N} q_{k} t^{k} s^{N-k}, \quad \bar{Q}(t)=\bar{Q}(t, 1), \quad \bar{P}(t)=t^{M} \bar{Q}(t)
$$

where $q_{0}, \ldots, q_{N-1} \in C, q_{N}=1, M$ is a non-negative integer. If there exists an $R \in R_{D} \cap V(X)$ (i.e. $R$ is a Volterna right inverse of $D$ ), then $\bar{P}(D) \in R(X)$ and the operator

$$
R_{0}=R^{M+N}(\bar{Q}(I, R))^{-1}
$$

is a Volterra right inverse of $\bar{P}(D)$.

Theorem 2 (von Trotha). If $R_{0}$ of the form (1) is a Volterra operator, then $R$ is Volterra.

We shall deal with $r$-Volterra operators and generalize Theorems 1 and 2 to the case of $\bar{Q}(t, s)$ being a polynomial with algebraic operator coefficients (cf. also [3], section 6). 
Definition 1. An operator $R \in L_{0}(X)$ is said to be $r$-Volterra if the operator $I-\lambda R$ is right invertible for all $\lambda \in C$.

We denote by $V_{r}(X)$ the set of all $r$-Volterra operators acting in $X$.

Lemma 1. Let $D \in R(X)$ and $R \in R_{D} \cap V_{r}(X)$. Then $\bar{Q}(I, R) \in R(X)$ and

$$
\bar{R}_{0}=R^{M+N} T, \quad \text { where } T \in R_{\bar{Q}(I, R)},
$$

is a right inverse of $\bar{P}(D)$.

P roof. Rewrite $\bar{Q}(I, R)$ in the form

$$
\bar{Q}(I, R)=\prod_{i=1}^{n}\left(I-t_{i} R\right)^{\alpha_{i}}, \quad \alpha_{1}+\ldots+\alpha_{n}=N .
$$

This implies that $\bar{Q}(I, R) \in R(X)$, provided that $R \in V_{r}(X)$. For every $T \in R_{\bar{Q}(I, R)}$, we have

$$
\bar{Q}(D) R^{N} T=\sum_{k=0}^{N} q_{k} D^{k} R^{N} T=\sum_{k=0}^{N} q_{k} R^{N-k} T=\bar{Q}(I, R) T=I .
$$

Consequently, the operator $\bar{P}(D):=D^{M} \bar{Q}(D)$ is also right invertible and

$$
\bar{P}(D) \bar{R}_{0}=D^{M} \bar{Q}(D) R^{M} R^{N} T=\bar{Q}(D) R^{N} T=I .
$$

LEMMA 2. If $\bar{R}_{0}$ of the form (2) is a $r$-Volterra operator, then $R$ is $r$-Volterra.

Proof. Let $s_{0}, \lambda \in C\left(\lambda \neq 0, s_{0} \neq 0\right)$ be fixed, $\mu=\bar{Q}(\lambda) \bar{s}_{0}^{M}$. It is easy to see that

$$
I-\mu \bar{R}_{0}=\bar{P}(D) R_{0}-\mu \bar{R}_{0}=\left(\bar{Q}(I, R)-\mu R^{N+M}\right) T .
$$

It follows that $\bar{Q}(I, R)-\mu R^{M+N}$ is right invertible, since $I-\mu R_{0}$ is right invertible.

Write: $\bar{P}_{\mu}\left(t, s_{0}\right)=\bar{Q}\left(t, s_{0}\right)-\mu s_{0}^{M+N}$.

We find

$$
\begin{aligned}
\bar{P}_{\mu}\left(\lambda s_{0}, s_{0}\right) & =\bar{Q}\left(\lambda s_{0}, s_{0}\right)-\mu s_{0}^{M+N} \\
& =\sum_{k=0}^{N} q_{k} \lambda^{k} s_{0}^{k} s_{0}^{N-k}-\mu s_{0}^{M+N}=s_{0}^{N}\left(\bar{Q}(\lambda)-\mu s_{0}^{M}\right)=0 .
\end{aligned}
$$

Hence

$$
\bar{P}_{\mu}\left(t, s_{0}\right)=\left(t-\lambda s_{0}\right) \bar{Q}_{\mu}\left(t, s_{0}\right),
$$

where $\bar{Q}_{\mu}\left(t, s_{0}\right)$ is a well-defined polynomial. 
Since $\bar{P}_{\mu}(I, R)=\bar{Q}_{\mu}(I, R)-\mu R^{M+N}$ is right invertible, we conclude that $I-\lambda R$ is right invertible for all $\lambda \in C$, i.e. $R \in V_{r}(X)$.

Now we deal with the case of polynomials in algebraic operators.

Let $D \in R(X), R \in R_{D}$ and let $A_{0}, \ldots, A_{N-1}, A_{N}=I$ be mutually commutative algebraic operators. Suppose that

$$
D A_{i}=A_{i} D \quad \text { on } \operatorname{dom} D ; \quad R A_{i}=A_{i} R \quad(i=0, \ldots, N) .
$$

Write

$$
Q(t, s)=\sum_{i=0}^{N} A_{i} t^{i} s^{N-i}, \quad Q(t)=Q(t, 1), \quad P(t)=t^{M} Q(t)
$$

( $M$ is a non-negative integer).

Theorem 1. Let $D \in R(X)$ and let $R \in R_{D} \cap V_{r}(X)$. Then $Q(I, R)$ is right invertible and $R_{0}=R^{M+N} T$ is a right inverse of $P(D)$ for every $T \in R_{Q(I, R)}$.

Proof. Write $\widehat{X}_{0}=\operatorname{lin}\left\{R^{k} ; k=0,1, \ldots\right\}$. Then $\widehat{X}_{0}$ is a commutative subalgebra in $L_{0}(X)$ and $Q(I, R)$ is a generalized algebraic element with characteristic roots belonging to the set (cf. Theorem 3.4 in [3]):

$$
\left\{I+\sum_{i=1}^{N} t_{n-i, k} R^{i},\left(i=1, \ldots, N ; k=1, \ldots, r_{N-1}\right)\right\},
$$

where $\left\{t_{i, 1}, \ldots, t_{i, r_{i}}\right\}$ are the characteristic roots of $A_{i}$. Lemma 1 implies that every operator belonging to the set $(5)$ is right invertible. This implies that $Q(I, R)$ is right invertible. If $T \in R_{Q(I, R)}$, then

$$
Q(D) R^{N} T=\sum_{i=0}^{N} A_{i} D^{i} R^{N} T=\sum_{i=0}^{N} A_{i} R^{N-i} T=Q(I, R) T=I .
$$

Consequently, the operator $P(D):=D^{M} Q(D)$ is right invertible and has a right inverse of the form $R_{0}=R^{M+N} T$.

Lemma 3. Let $D \in R(X)$ and $R \in R_{D} \cap V_{r}(X)$. If $A$ is an algebraic operator and $A R=R A$, then $R A$ is a $r$-Volterra operator.

Proof. Since $I+\lambda R A$ is a generalized algebraic operator over $\widehat{X}_{0}$ for every $\lambda \in C$ with characteristic roots of the form $I+\lambda t_{i} R$ (where $t_{i}$ $(i=1, \ldots, N)$ are characteristic roots of the operator $A)$, it follows that $I+\lambda A R$ is right invertible for all $\lambda \in C$, i.e. $A R$ is $r$-Volterra operator.

A converse result to Theorem 1 is the following 
Theorem 2. Let $D \in R(X), R \in R_{D}$ and let $A_{0}, \ldots, A_{N}$ be algebraic operators satisfying the conditions (3). Let $Q(t, s), Q(t)$ and $P(t)$ be defined by (4). If $Q(I, R)$ is right invertible, then

$$
R_{0}:=R^{M+N} T \in R_{P(D)} \quad \text { for every } T \in R_{Q(I, R)} .
$$

Moreover, if $A_{i} T=T A_{i}(i=1, \ldots, N)$ and $R_{0} \in V_{r}(X)$, then $R \in V_{r}(X)$.

P roof. It is enough to check that $R \in V_{r}(X)$ provided that $R_{0} \in V_{r}(X)$. Let $s_{0}, \beta \in C$ be fixed.

Write: $A=Q(\beta) s_{0}^{-M}$. Clearly, $A$ is an algebraic operator and $A R_{0}=$ $K_{0} A$. Lemma 3 implies that $A R_{0}$ is a $r$-Volterra operator. Hence, $I-A R_{0}$ is right invertible.

On the other hand, $I-A R_{0}=\left(Q(I, R)-A R^{M+N}\right) T$. If $S \in R_{I-A R_{0}}$ then $I=\left[Q(I, R)-A R^{M+N}\right] T S$. It means that the operator $Q_{0}:=Q(I, R)-$ $A R^{M+N}$ is right invertible. Write

$$
H_{A}\left(t, s_{0}\right)=Q\left(t, s_{0}\right)-A s_{0}^{M+N} .
$$

Then we have

$$
\begin{aligned}
H_{A}\left(\beta s_{0}, s_{0}\right) & =Q\left(\beta_{s_{0}}, s_{0}\right)-A s_{0}^{M+N} \\
& =\sum_{k=0}^{N} q_{k} \beta^{k} s_{0}^{k} s_{0}^{N-k}-A s_{0}^{M+N}=s_{0}^{N}\left(Q(\beta)-A s_{0}^{M}\right)=0 .
\end{aligned}
$$

It implies

$$
H_{A}\left(t, s_{0}\right)=\left(t-\beta s_{0}\right) Q_{A}\left(t, s_{0}\right)
$$

Thus

$$
H_{A}(I, R)=(I-\beta R) Q_{A}(I, R) .
$$

The operator $H_{A}(I, R)$ is right invertible for all $\beta \in C$, which implies that $I-\beta R$ is right invertible for all $\beta \in C$, i.e. $R \in V_{r}(X)$.

Corollary 1. Let $D \in R(X), R \in R_{D}$, and let $A$ be an algebraic operator commuting with $R$. If $A R$ is an $r$-Volterra operator and $A$ is invertible, then $R$ is an $r$-Volterra operator.

Corollary 2. Let $D \in R(X), R \in R_{D} \cap V_{r}(X)$. Suppose that $A$ has the characteristic polynomial of the form

$$
P_{A}(t)=\prod_{j=1}^{n}\left(t-t_{j}\right), \quad t_{i} \neq t_{j} \text { for } i \neq j
$$

and $A R=R A$. 
Then every solution of the equation

$$
(D-A) x=y, \quad y \in X
$$

is of the form

$$
x=\sum_{i=1}^{n}\left[T_{i} P_{i}(R y+z)+c_{i}\right],
$$

where $T_{i} \in R_{I-t_{i} R}, z \in \operatorname{ker} D, c_{i} \in \operatorname{ker} T_{i}$ and

$$
P_{i}=\prod_{k=1, k \neq i}^{n}\left(t_{i}-t_{k}\right)^{-1}\left(A-t_{k} I\right) .
$$

Proof. Rewrite (6) in the form

$$
x-R A x=R y+z, \quad z \in \operatorname{ker} D .
$$

Since $A=\sum_{i=1}^{n} t_{i} P_{i},(8)$ is equivalent to the equation

$$
\left(I-\sum_{i=1}^{n} t_{i} R P_{i}\right) x=R y+z .
$$

Acting on both sides of (9) by the operator $P_{k}$, we obtain

$$
\left(I-t_{k} R\right) P_{k} x=P_{k}(R y+z), \quad(k=1, \ldots, n) .
$$

By our assumption $T_{k} \in R_{I-t_{k} R},(10)$ are equivalent to the following equations

$$
P_{k} x=T_{k} P_{k}(R y+z)+c_{k},
$$

where $c_{k} \in \operatorname{Ker}\left(I-t_{k} R\right), k=1,2, \ldots, n$. Thus

$$
x=\sum_{i=1}^{n}\left[T_{i} P_{i}(R y+z)+c_{i}\right] .
$$

The proof is complete.

\section{References}

[1] D. Przeworska-Rolewicz, S. Rolewicz, Equations in Linear Spaces, PWN, Warszawa, 1968.

[2] D. Przeworska-Rolewicz, Algebraic Analysis, PWN-Polish Scientific Publishers and D. Reidel Publishing Company, Warszawa-Dordrecht, 1988. 
[3] Nguyen Van Mau, Boundary value problems and controllability of linear systems with right invertible operators, Dissertationes Math. CCCXVI, Warszawa, 1992.

[4] $\mathrm{Nguyen} \mathrm{Van} \mathrm{Mau,} \mathrm{Generalized} \mathrm{Algebraic} \mathrm{Elements} \mathrm{and} \mathrm{Linear} \mathrm{Singular} \mathrm{Integral}$ Equations with Transformed Argument, Warsaw University of Technology, Warszawa, 1989.

FACULTY OF MATHEMATICS

HANOI UNIVERSITY

90 Nguyen Trai, Dong Da,

HANOI, VIETNAM

Received September 1st, 1994. 